\title{
Improved Evaluation of Wheat Cultivars (Lines) on Resistance to Puccinia striiformis f. sp. tritici Using Molecular Disease Index
}

\author{
Bingyao Chu, ${ }^{1}$ Lujia Yang, ${ }^{1}$ Cuicui Wang, ${ }^{1}$ Yilin Gu, ${ }^{1}$ Kai Yuan, ${ }^{1}$ Rui Wang, ${ }^{2}$ Yong Luo, ${ }^{1 \dagger}$ and Zhanhong $\mathrm{Ma}^{1 \dagger}$ \\ ${ }^{1}$ Department of Plant Pathology, Ministry of Agriculture and Rural Affairs (MOA) Key Lab of Pest Monitoring and Green \\ Management, College of Plant Protection, China Agricultural University, Beijing 100193, China; and ${ }^{2}$ Kaifeng Experimental \\ Station of China Agricultural University, Kaifeng 475004, China
}

\begin{abstract}
Wheat stripe rust caused by Puccinia striiformis f. sp. tritici is one of the most destructive diseases of wheat worldwide. Sichuan Province plays an important role in interregional epidemics in China. Application of host resistance is important in disease management, and efficient approaches to evaluate resistance level are necessary to obtain useful varieties. In this study, 100 wheat cultivars (lines) growing in Sichuan were selected to evaluate their resistance to stripe rust. Field experiments were conducted with a mixture of three P. striiformis $\mathrm{f}$. sp. tritici races for inoculations at seeding and adult stages in the 2014 to 2015 season and the 2016 to 2017 season. Leaf samplings were conducted four times during the latent period at early growth stage of wheat. The sampled leaves were processed to extract DNA. The

under the disease progress curve in terms of disease index (AUDPCDI) was obtained for each studied cultivar (line) in the fields. Among the 100 studied cultivars (lines), $61 \%$ of them showed seedling resistance, and 63 and $65 \%$ showed adult resistance in the 2014 to 2015 and 2016 to 2017 seasons, respectively, based on the infection type. High consistency in resistance grouping by cluster analysis as the percentage of the studied cultivar (line) belonging to the same group based on AUDPC-DI data and based on MDI data was obtained. The correlations between AUDPC-DI and MDI from samples collected on 9 and 14 or 15 days after inoculation during the latent period were all significant at $P<0.01$. This study provided a new and efficient method for evaluation of varietal resistance to wheat stripe rust.
\end{abstract} DNA of both wheat and $P$. striiformis $\mathrm{f}$. sp. tritici was quantified using real-time quantitative polymerase chain reaction, and the molecular disease index (MDI) was used to evaluate the resistance level. The area
Keywords: causal agent, cereals and grains, crop type, field crops, fungi, subject areas
Wheat stripe rust, caused by Puccinia striiformis f. sp. tritici, is one of the most important diseases worldwide (Chen 2005; Wellings 2007; Zeng and Luo 2006). China is the largest epidemic region of the disease (Stubbs 1988). The most destructive epidemics of stripe rust in China occurred in 1950, 1964, 1990, and 2002, causing yield losses of 60, 30, 18, and 13 hundred million tons, respectively (Wan et al. 2004). Host resistance and chemical control are the two main strategies for disease management (Wan et al. 2007). However, the new biological pathogenic type V26 was first discovered in Sichuan Province in 2008 to 2009. This biological pathogenic type could infect wheat cultivars (lines) with $\mathrm{Yr} 26$ and $\mathrm{YrCH} 42$ genes (Liu et al. 2010). As the epidemics of new P. striiformis f. sp. tritici race, more wheat cultivars (lines) are at risk of resistance loss. Understanding the situation of resistance of main wheat cultivars (lines) planted in Sichuan Province is of importance.

One of the conventional ways to evaluate host resistance is to score the infection type (IT) based on visual symptoms. For wheat stripe rust, two scales are normally used: one scale from one to nine (McNeal et al. 1971) and another scale from one to four (Stubbs 1985). Because resistance can be expressed differently at various growth stages, such as seedling resistance, adult resistance, or allstage resistance, evaluation of resistance level only at one growth stage likely cannot represent real situations of resistance expressed

${ }^{\dagger}$ Corresponding authors: Y. Luo; ygluo@ucanr.edu, and Z. Ma; mazh@cau.edu.cn

Funding: This study was supported by National Key Research and Development Program of China Grants 2017YFD0200400 and 2017YFD0201700 and Key Research and Development Projects of Ningxia Hui Autonomous Region East and West Science and Technology Cooperation Project.

The author(s) declare no conflict of interest.

Accepted for publication 23 October 2018.

() 2019 The American Phytopathological Society in the fields. It is essential to consider resistance expression in the entire growing season to evaluate host resistance, especially for the cultivars expressed as horizontal resistance or durable or tolerant resistance. However, it is difficult to perform this kind of evaluation method for large numbers of cultivars or breeding lines. Therefore, efficient methods to quickly and accurately evaluate host resistance levels are needed. Such methods should be applied to approximately evaluate the true situation and potential of resistance level at an accepted variation or error range statistically.

The real-time quantitative polymerase chain reaction (qPCR) could be an alternative approach in host resistance assessment and quantification. Unlike conventional polymerase chain reaction (PCR) that uses an end point analysis of the amplicon, qPCR detects and quantifies PCR products by using either fluorescent-specific probes (such as TaqMan and molecular beacon) or double-stranded DNA binding fluorescent dyes (such as ethidium bromide and SYBR green I) (Wilhelm and Pingoud 2003). qPCR is widely used in detection and quantification of target DNA of plant pathogenic microorganisms from soil (Cullen et al. 2001; Filion et al. 2003; Luo et al. 2009), air (Luo et al. 2007; Schweigkofler et al. 2004), seeds (McNeil et al. 2004), and plant tissues (Brouwer et al. 2003; Gachon and Saindrenan 2004; Mercado-Blanco et al. 2003; Sandberg et al. 2003; Waalwijk et al. 2004). Concerning the evaluation of cultivar resistance, rice pathogen Magnaporthe grisea (Qi and Yang 2002), alfalfa pathogen Phytophthora medicaginis (Vandemark and Barker 2003), oat pathogen Puccinia coronata f. sp. Avenae (Jackson et al. 2006), and bean pathogen Fusarium oxysporum f. sp. phaseoli (Xue et al. 2012) were quantified in plant tissues of different varieties, and different levels of resistance were clearly discriminated using qPCR. For detection of wheat stripe rust, Yan et al. (2012) applied qPCR with the method of SYBR green I to quantify $P$. striiformis f. sp. tritici in wheat leaves during the latent period in the fields, showing significant potential of prediction for the future disease development.

The above research provided efficient and potential approaches for evaluation of resistance of different cultivars with molecular methods that overcome the deficiency of the conventional methods. 
Moreover, the expression of host resistance at very early growth stages could not be likely visualized, such as during the latent period. The qPCR method could be applied to obtain the significant signal on how the host reacts to the pathogen infection. This study attempted to illustrate the feasibility and advantages of using such a method to evaluate resistance levels to wheat stripe rust.

Development of wheat stripe rust has a feature that the period of latent infection could take from several days to even several months depending on growing conditions and environment, especially the temperature. In the field, the shortest latent period is normally 8 to 10 days in the spring, whereas the longest latent period could last $>60$ days in the autumn and winter (Xiao et al. 1983; Zeng et al. $1981 \mathrm{~b})$. The reaction process of the host to the pathogen infection during this period of time could contain important information on the host $\times$ pathogen interaction, which will be useful in evaluation of host resistance. However, such information is difficult to obtain because of the nonvisualization feature in the process. The development of disease is a progress of interaction between host and pathogen that may happen in different ways, such as the four phases of plant pathogen interaction in the zigzag model consisting of pathogen-associated molecular pattern (PAMP)-triggered immunity, effector-triggered susceptibility, effector-triggered immunity (ETI), and the repeat of ETI (Jones and Dangl 2006). However, the relationship of such processes with the corresponding amount of pathogen in the plant tissues as well as the future disease expression on different cultivars in the field are still under study. Using qPCR could help us understand more about such interactions to efficiently evaluate the resistance level that may contribute to disease management.

Wheat stripe rust is a polycyclic disease, and the area under the disease progress curve in terms of disease index (AUDPC-DI) from multiple disease assessments could represent the actual resistance level of different cultivars (lines) expressed in the fields. The molecular disease index (MDI), quantified as the proportion of $P$. striiformis f. sp. tritici DNA (picograms) over the total amount of wheat DNA (nanograms), could reflect infection level of $P$. striiformis $\mathrm{f}$. sp. tritici in the wheat leaves at a certain time during the latent period (Yan et al. 2012). The objectives of this study were to (i) evaluate the resistance of the 100 main wheat cultivars (lines) planted in Sichuan Province with conventional methods, (ii) obtain the MDI values for these cultivars (lines), and (iii) analyze the relationship between AUDPC-DI and MDI to evaluate the usefulness of the method that we generated in varietal resistance assessment.

\section{Materials and Methods}

Plant and pathogen materials. One hundred wheat cultivars (lines) were provided by the Southwest University of Science and Technology in China. These cultivars (lines) were mainly planted in Sichuan Province in recent years. The universally susceptible variety Mingxian 169 was obtained from the Plant Disease Epidemiology Laboratory, Department of Plant Pathology, China Agricultural University. The Chinese predominant $P$. striiformis $\mathrm{f}$. sp. tritici physiological races CYR32 and CYR33 and pathogenic type G22-83 (Huang et al. 2014; Liu et al. 2017) were used in this study for all test assays.

Resistance evaluation with the conventional method. The seeds of all of the 100 cultivars (lines) were soaked in sterile water for $10 \mathrm{~h}$ and split into 25 groups. Six to seven seeds of each of the four cultivars (lines) of each group were sown in a pot $(10 \times 10 \times 10 \mathrm{~cm})$, and Mingxian 169 was sown as the susceptible control. The potted plants were grown in a growth chamber at 10 to $15^{\circ} \mathrm{C}$ with 60 to $70 \%$ relative humidity and a 14/10-h light/dark photoperiod (10,000 lux). When the first leaves of seedlings were fully expanded, leaf surfaces were rubbed to remove wax, sprayed with appropriate amount of water, and inoculated with $P$. striiformis f. sp. tritici mixed races (CYR32:CYR33:pathogenic type G22-83 by 1:1:1) with $1 \mathrm{ml}$ spore suspension at the concentration of $0.5 \mathrm{mg} / \mathrm{ml}$. The inoculated seedlings were incubated at 8 to $10^{\circ} \mathrm{C}$ with dew for $24 \mathrm{~h}$ in darkness to promote infection and then transferred to the growth chamber for 18 days under the same condition described above. When Mingxian 169 showed disease symptoms, a system of a zero to four scale
(Stubbs 1985) was used to assess IT of the inoculated seedlings. Three replicated pots were used in the test.

A field experiment was conducted in Xinghuaying Town $\left(114.26^{\circ}\right.$ E and $34.76^{\circ} \mathrm{N}$ ), Henan Province, China in 2014 to 2015 and 2016 to 2017. The soil type was sandy loam. Each cultivar (line) was planted in a $1-\mathrm{m}$ row with $0.25-\mathrm{m}$ spacing between two rows. The study was performed with three replicates, and Mingxian169 was used as a susceptible control. Inoculations were conducted on 23 March 2015 and 13 March 2017. Urediniospores of races CYR32, CYR33, and $P$. striiformis $\mathrm{f}$. sp. tritici-G22-83 were mixed with the equal proportion to the concentration of $80 \mathrm{mg} /$ liter. Each cultivar (line) was inoculated by spraying $20 \mathrm{ml}$ of spore suspension and then covered with plastic sheets to maintain high humidity overnight. Disease was assessed five times at 3- to 5-day intervals after Mingxian169 showed symptoms. The first time of disease recording was conducted on 7 April 2015 and 1 April 2017. For each tested cultivar (line), 25 plants (about 100 leaves) were randomly selected to record IT and number of diseased leaves to calculate incidence and disease severities. Disease severity was assessed as the percentage of lesion area over the whole leaf area (Shang et al. 1990). Wheat growth stages were also recorded according to Zadoks et al. (1974). Disease index (DI) was calculated as the average disease severity $\times$ the disease incidence $\times 100$, and the mean DI for each cultivar (line) was obtained from three replicates.

Leaf sampling and DNA extraction. In the field experiment, leaf samplings were conducted four times during the latent period in 2015 and two times in 2017. The samplings were arranged 1, 5, 9, and 14 days after inoculation (DAI) in 2015 and 9 and 15 DAI in 2017, respectively. On each sampling date, 30 leaves were randomly collected from each replicate of each cultivar (line). The Cetyl trimethy-lammonium bromide (CTAB) method (Enjalbert et al. 2002), with minor modification (Pan et al. 2010; Zeng et al. 2010), was applied to obtain DNA for each sample.

Quantification of pathogen using real-time PCR. Duplex realtime qPCR amplifications were performed using the TaqMan method. The previously published primer pairs specific to wheat and $P$. striiformis f. sp. tritici (Pan et al. 2016) and the probes specific to wheat and $P$. striiformis $\mathrm{f}$. sp. tritici were used. The following primer pair specific to $P$. striiformis was used: $P$. striiformis $\mathrm{f}$. $\mathrm{sp}$. tritici-F ( $5^{\prime}$-GGATGTTGAGTGCTGCTGTAA-3')/P. striiformis f. sp. tritici-R (5'-TTGAGGTCTTAAGGTTAAAATTG-3') with the $P$. striiformis f. sp. tritici-specific probe $P$. striiformis f. sp. triticiPro (5'-TCACATCAAGGATTGTAGCAATACTGCCA-3'). The following wheat-specific primer pair was used: TAG2315F (5'CAGAAAGCGAGTGGAAAGATGAAAG-3')/TAG2473R (5'-GCAA GGAGGACAAAGATGAGGAA-3') with the wheat-specific probe TAG-Pro (5'-CAAGCATCAAAGGCAAGCAAGCAGTAGT-3') (Pan et al. 2016). Amplifications were conducted in a $20-\mu 1$ volume

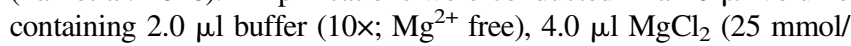
liter), $2.0 \mu \mathrm{l}$ deoxyribonucleotide triphosphate (dNTP) ( $2.5 \mathrm{mmol} / \mathrm{liter}$ ), $0.4 \mu \mathrm{l}$ each primer (10 $\mu \mathrm{mol} / \mathrm{liter}), 0.3 \mu \mathrm{l}$ each probe $(10 \mu \mathrm{mol} / \mathrm{liter}), 0.4 \mu \mathrm{l}$ Taq polymerase $(5 \mathrm{U} / \mu \mathrm{l}), 2.0 \mu \mathrm{l}$ template DNA, and $7.6 \mu 1 \mathrm{ddH}_{2} \mathrm{O}$. The reaction condition was initiated with denaturation at $94^{\circ} \mathrm{C}$ for $3 \mathrm{~min}$ followed by 40 cycles of denaturation at $94^{\circ} \mathrm{C}$ for $20 \mathrm{~s}$, annealing at $57^{\circ} \mathrm{C}$ for $30 \mathrm{~s}$, and extension at $72^{\circ} \mathrm{C}$ for $20 \mathrm{~s}$. The fluorescence signal from the probe was recorded at $72^{\circ} \mathrm{C}$ during each cycle.

To quantify the DNA amounts of both wheat and P. striiformis $\mathrm{f}$. sp. tritici in each reaction, standard curves for $P$. striiformis f. sp. tritici and wheat were established. The DNA of urediniospores of $P$. striiformis f. sp. tritici and wheat leaves was extracted using the CTAB method as described above. The concentrations of both $P$. striiformis f. sp. tritici and wheat DNAs were determined with Denovix (DS-11 Spectrophotometer). The $P$. striiformis f. sp. tritici DNA was 10 times diluted from 44 to $0.00044 \mathrm{pg} / \mu \mathrm{l}$, whereas wheat DNA was 10 times diluted from 525 to $0.0525 \mathrm{ng} / \mu$ l. These diluted DNA series were used in $\mathrm{qPCR}$ following the PCR conditions described above with five replicates. The corresponding cycle threshold $(\mathrm{Ct})$ values of $P$. striiformis f. sp. tritici and wheat were recorded. The standard curve of $P$. striiformis $\mathrm{f}$. sp. tritici described as the $\log$ DNA concentration (picograms; fluorescein amidite [FAM]) versus 
corresponding Ct value was generated. Similarly, the standard curve of wheat described as the log DNA concentration (nanograms; hexachlorofluorescein $[\mathrm{HEX}]$ ) versus the corresponding $\mathrm{Ct}$ value was also generated.

For each sample in each qPCR reaction, $P$. striiformis $\mathrm{f}$. sp. tritici and wheat DNA was used as the positive control, and $\mathrm{ddH}_{2} \mathrm{O}$ was used as the negative control. Three replicates were used for each sample, and the mean $\mathrm{Ct}$ value was calculated. MDI (Yan et al. 2012) was calculated for each replicate of each cultivar (line) as MDI $=$ quantity of $P$. striiformis $\mathrm{f}$. sp. tritici DNA in picograms/ quantity of wheat DNA in nanograms $\times 1,000$, and the mean
MDI for each cultivar (line) was calculated from three replicates for analysis.

Data analysis. In the field experiment, the AUDPC-DI covering five times the disease assessments at different periods was calculated for each replicate of each cultivar (line) using the following formula:

$$
\text { AUDPC-DI }=\sum_{i}^{n}\left[\left(\frac{x_{i}+x_{i+1}}{2}\right)\left(t_{i+1}-t_{i}\right)\right]
$$

where $n$ is the total number of disease assessments; $x_{i}$ and $x_{i+1}$ are the DIs at $i$ th and $(i+1)$ th disease assessments, respectively; and

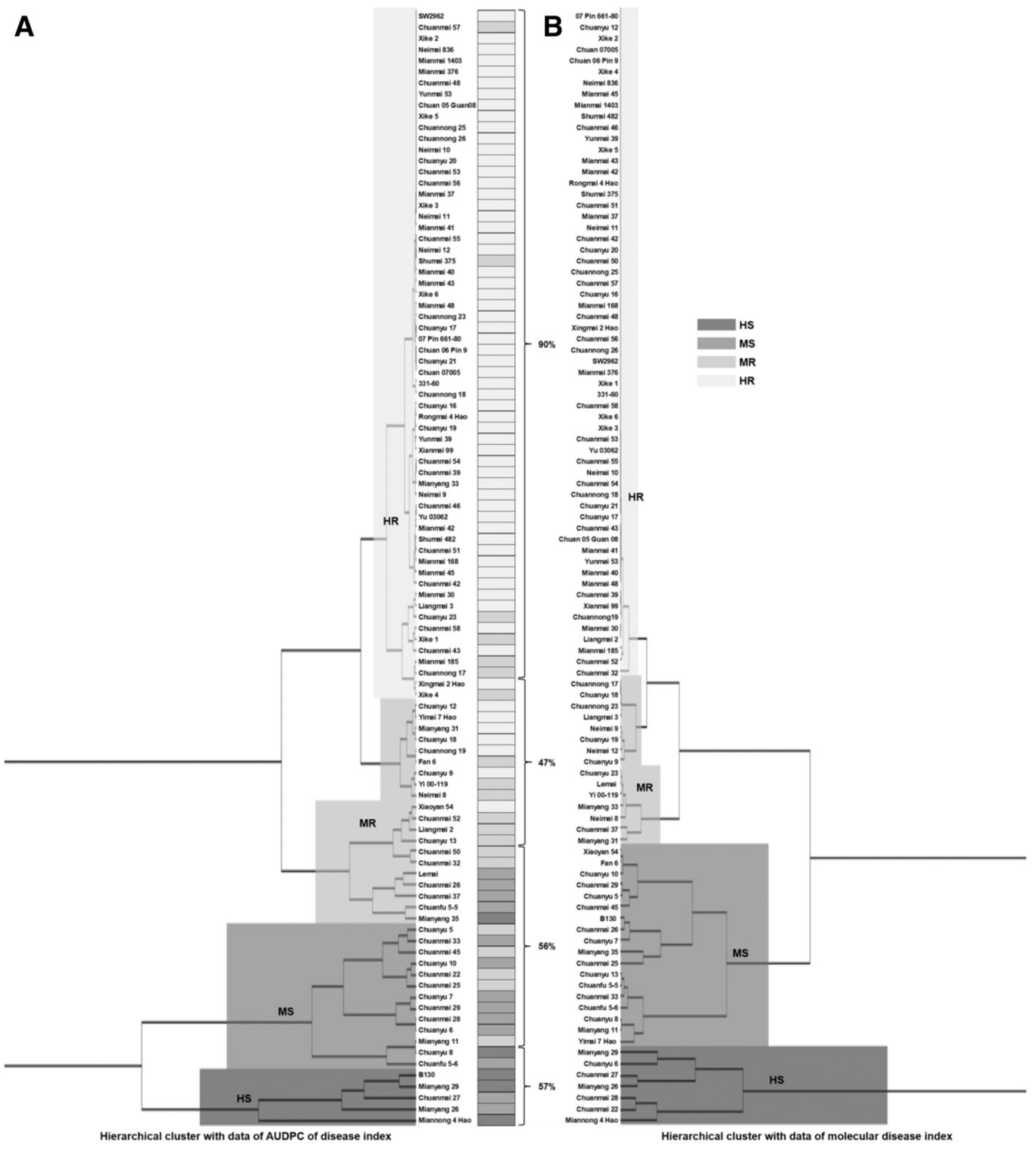

Fig. 1. Hierarchical cluster analysis for 100 cultivars (lines) tested in this study using the area under the disease progress curve in terms of disease index (AUDPC-DI) data (A) and the molecular disease index data (B) of 14 days after inoculation leaf sampling in 2014 to 2015 . HR, highly resistant to wheat stripe rust; HS, highly susceptible to wheat stripe rust; MR, moderately resistant to wheat stripe rust; MS, moderately susceptible to wheat stripe rust. 
$t_{i}$ and $t_{i+1}$ are days of the disease progress curve at $i$ th and $(i+1)$ th disease assessments, respectively.

To determine the consistency in resistance grouping of 100 cultivars (lines) between the method using AUDPC-DI data and that using MDI data, cluster analyses were conducted with the dataset of
AUDPC-DI and the dataset of MDI, respectively, of 14 and 15 DAI for these tested cultivars (lines) using hierarchical clustering with the method of "average" based on the "Euclidean" distance with R Studio (RStudio X64 3.4.1) in 2 years. The library of ggtree in R was used to highlight the four different resistance groups as highly

A

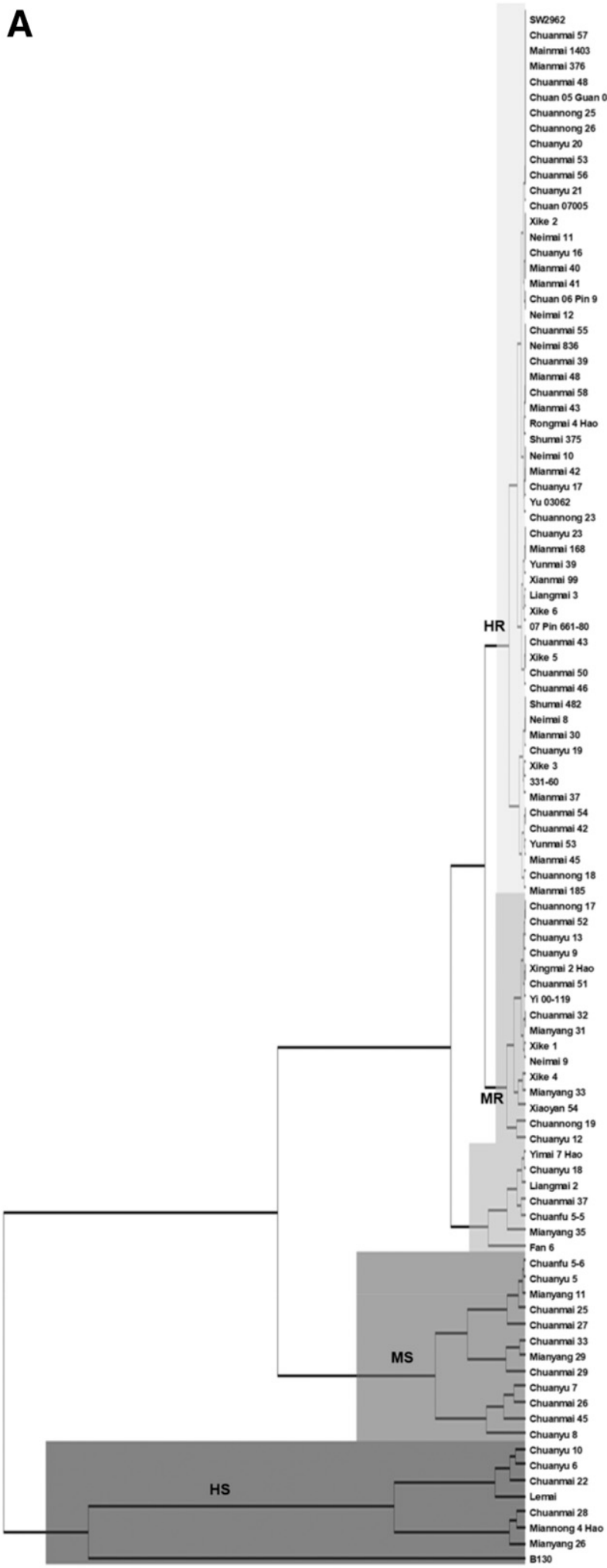

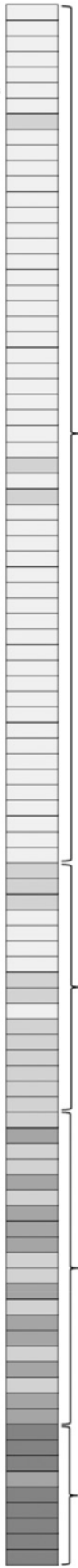

B

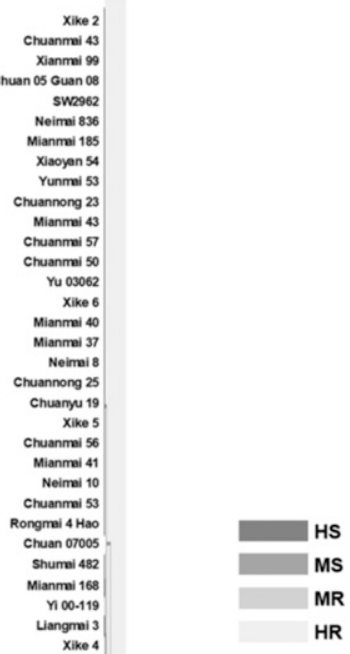

Neimal 12

\begin{tabular}{c} 
Mianmmi 48 \\
Chuannmal 55 \\
\hline
\end{tabular}

Chuanmai 39

07 Pin $661 .-80$

\begin{tabular}{r|r} 
Chuangu 17 & 17 \\
Chuangu 20 & HR \\
chuanmi 54 &
\end{tabular}

(n)

Xike 3

Shumi i 375

Mianmei 42

Miannmel 376

Chuarny 21

Chuannong 26
Chuannong 18

Yunnail 39

Chuaryu 23

Chuaryu 16

Chuanmei 46

Mianmai 1403.

Chuan $06 \mathrm{Pin} 9^{\circ}$

nos Ping

. Chuanmei 51 .

Xike 1
$331-50$

(1)

Mianmie 45 -

Mianmei 30

Xingmai 2 Hao
Chuanmei 32

Chuanmei 42, MR

Chuarny 18 Chuanyu 9 .

Manyang

Chuanmei 52 .

-

Chuaryu 12 .

Chuanmia 29

Mianyang 35

Mianyang 33

Mianyang 20

uannong 17

chuanfu 5-6

Chuanyus 8

Chuanmial 33

Chuaryu 13

Yimai 7 Hao

Chyanneit 37

Chuanmei 25

Miarrang 11 -

Chuantu 5.5 -

Chuanmei 27

Fan 6

Chuanyu 7

Chuanye 5 -
Lemai -

Chuaryu 10

Chuanyu 6

Chuanmai 26

Chuanmí 22

Chuanmei 20

Mianyang 26
mnong 4 HaO

B130

130

Fig. 2. Hierarchical cluster analysis for 100 cultivars (lines) tested in this study using the area under the disease progress curve in terms of disease index (AUDPC-DI) data (A) and the molecular disease index data (B) of 15 days after inoculation leaf sampling in 2016 to 2017 . HR, highly resistant to wheat stripe rust; HS, highly susceptible to wheat stripe rust; MR, moderately resistant to wheat stripe rust; MS, moderately susceptible to wheat stripe rust. 
susceptible, moderately susceptible, moderately resistant, and highly resistant based on the results of cluster.

To determine the relationship between AUDPC-DI and MDI from different periods of sampling times, AUDPC-DI and corresponding MDI of each of the 100 cultivars (lines) were used in correlation analyses performed with liner regression analyses of SAS (version 9.4; SAS Institute, Cary, NC). The 2014 to 2015 data included AUDPC-DI and MDIs data of 1, 5, 9, and 14 DAI, and the 2016 to 2017 data included AUDPC-DI and the MDIs data of 9 and 15 DAI.

\section{Results}

Field evaluation of host resistance. The IT data of 100 cultivars (lines) at the seeding (growth stage [GS] $=11$ to 12 ) and adult (GS = 39 to 45) (Zadoks et al. 1974) stages were obtained. Of the 100 cultivars (lines), 61 showed seedling resistance, and 63 and 65 showed adult resistance in the 2014 to 2015 and 2016 to 2017 seasons, respectively. There were 48, 15, 13, and 24 cultivars (lines) that were all-stage resistant, adult resistant, seeding resistant, and all-stage susceptible, respectively, from the 2014 to 2015 experiment. There were $49,16,12$, and 23 cultivars (lines) that were all-stage resistant, adult

Table 1. Resistance grouping of 100 wheat cultivars (lines) ${ }^{\mathrm{a}}$

\begin{tabular}{lccccc}
\hline Year & HS, \% & MS, \% & MR, \% & HR, \% & All, \% \\
\hline $2014-2015$ & 57 & 56 & 47 & 90 & 75 \\
$2016-2017$ & 89 & 55 & 69 & 95 & 82 \\
\hline
\end{tabular}

${ }^{a}$ Four groups were considered, and a total of 100 wheat cultivars (lines) were studied for their resistance levels to stripe rust. HR, highly resistant to wheat stripe rust; HS, highly susceptible to wheat stripe rust; MR, moderately resistant to wheat stripe rust; MS, moderately susceptible to wheat stripe rust. resistant, seeding resistant, and all-stage susceptible, respectively, from the 2016 to 2017 experiment.

The 100 cultivars (lines) were classified into four groups with the hierarchical cluster analysis based on AUDPC-DI data. In the 2014 to 2015 experiment, 5 cultivars (lines) were highly susceptible, 13 were moderately susceptible, 20 were moderately resistant, and 62 were highly resistant or immune (Fig. 1A). In the 2016 to 2017 experiment, 8 cultivars (lines) were highly susceptible, 12 were moderately susceptible, 23 were moderately resistant, and 57 were highly resistant or immune (Fig. 2A). Among highly susceptible, moderately susceptible, moderately resistant, and highly resistant or immune cultivars (lines), 3, 9, 16, and 56, respectively, were consistent between the 2 years of results, and 16 cultivars (lines) behaved as different resistance levels. After combining IT and cluster results for the tested cultivars (lines), most of them were resistant to Chinese predominant stripe rust races CYR32, CYR33, and pathogenic type G22-83.

Standard curves of $\boldsymbol{P}$. striiformis $\mathbf{f}$. sp. tritici and wheat. The equation to quantify the DNA amount of $P$. striiformis $\mathrm{f}$. sp. tritici was $y_{1}=-0.2947 x_{1}+6.1805\left(R^{2}=0.9925, P<0.01\right)$, where $y_{1}$ is $\log _{10}$ value of the concentration of $P$. striiformis f. sp. tritici's DNA in picograms and $x_{1}$ is the corresponding $\mathrm{Ct}$ value. The equation to quantify the DNA of wheat was $y_{2}=-0.2979 x_{2}+8.5922$ $\left(R^{2}=0.9755, P<0.01\right)$, where $y_{2}$ is the $\log _{10}$ value of the concentration of wheat's DNA in nanograms and $x_{2}$ is the corresponding $\mathrm{Ct}$ value.

Cluster analysis. Using 2 years of data of MDIs of 14 or 15 DAI, the 100 cultivars (lines) were classified into four groups by the hierarchical cluster analysis. Based on the 2014 to 2015 experiment, 7 cultivars (lines) were highly susceptible, 18 were moderately susceptible, 15 were moderately resistant, and 60 were highly resistant or immune (Fig. 1B). Based on the 2016 to 2017 experiment, 9 cultivars
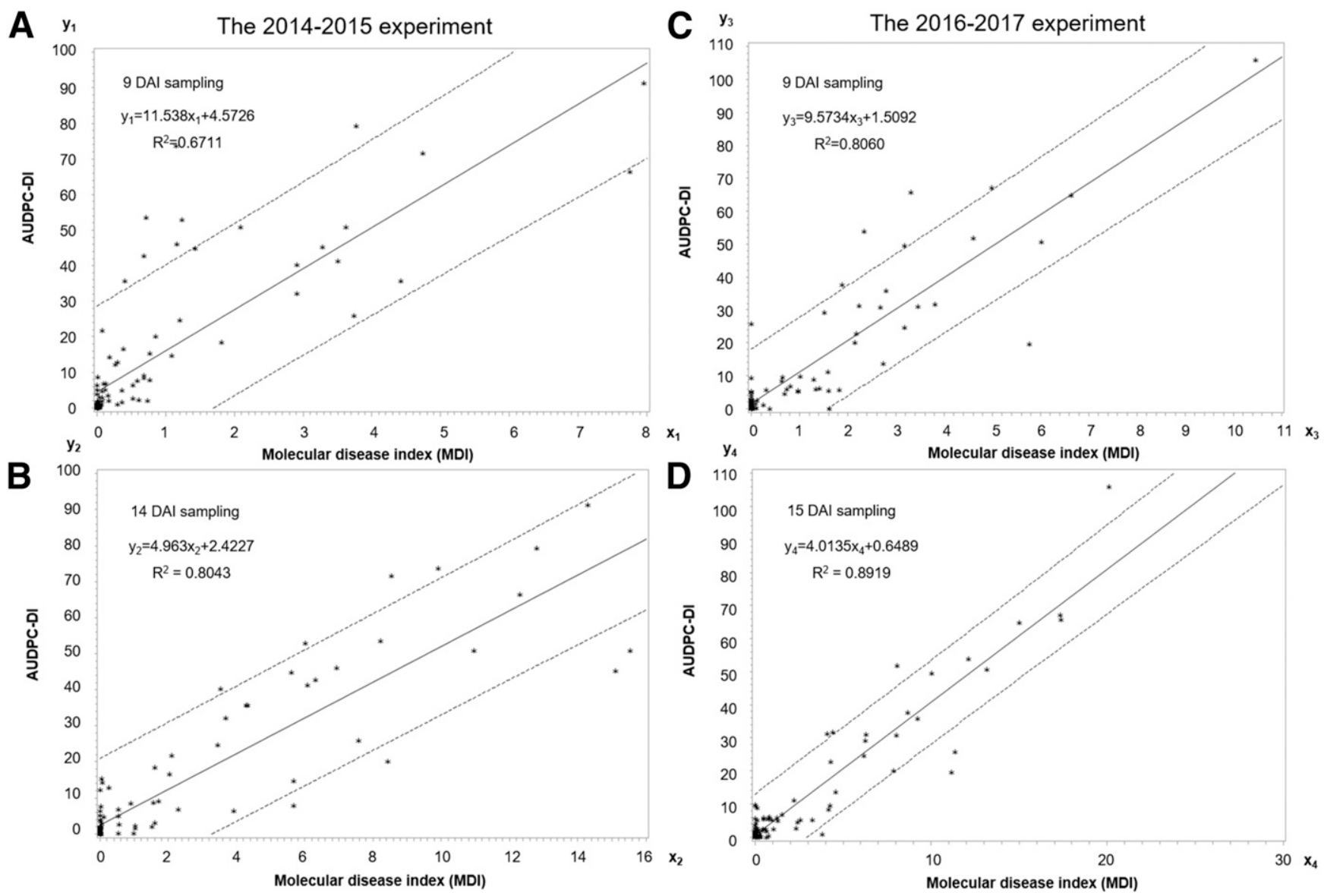

Fig. 3. Linear regressions of area under the disease progress curve in terms of disease index (AUDPC-DI) with molecular disease index (MDI) of 9 days after inoculation (DAI) of sampling (A) and MDI of 14 DAl of sampling (B) in the 2014 to 2015 experiment and linear regressions of AUDPC-DI with MDI of 9 DAl of sampling (C) and MDI of 15 DAl of sampling (D) in the 2016 to 2017 experiment. The dotted lines represented 95\% confidence intervals. 
(lines) were highly susceptible, 20 were moderately susceptible, 16 were moderately resistant, and 55 were highly resistant or immune (Fig. 2B).

Intensive analysis was performed to determine the percentage of the tested cultivars (lines) that belonged to the same group of clusters based on AUDPC-DI data and MDI data. The results of clustering analysis based on AUDPC-DI data were used as the reference, and the percentage of cultivars (lines) clustered based on MDI data that matched to the same group based on the reference was calculated for each of the four groups. In the 2014 to 2015 experiment for the highly susceptible cultivar (line) group, which had seven cultivars (lines) clustered based on MDI data, four cultivars were attributed as the same group and three cultivars were clustered to the moderately susceptible group based on the AUDPC-DI data. Similarly for the group of moderately susceptible cultivars (lines), which had 18 cultivars (lines), 10 were attributed to the same group, 7 were attributed to the moderately resistant group, and 1 (B130) was attributed to the highly susceptible cultivar group based on the AUDPCDI data. For the group that had 15 moderately resistant cultivars (lines), 7 were attributed to the same group and 8 were attributed to the highly resistant cultivar group based on the AUDPC-DI data. Among 60 highly resistant cultivars (lines), 54 cultivars were attributed to the same group and 6 cultivars were clustered to the moderately resistant group based on AUDCP-DI data. The 2016 to 2017 experiment showed that $89,55,69$, and $95 \%$ of cultivars (lines) clustered based on the MDI data belonged to the same groups clustered based on AUDCP-DI data for highly susceptible, moderately susceptible, moderately resistant, and highly resistant groups, respectively (Table 1). The comparison showed the high consistency of such percentages between the 2 years' results for the moderately susceptible and highly resistant groups and some levels of variation of such percentages for the highly susceptible and moderately resistant groups (Table 1).

Correlation between AUDPC-DI and MDI. All correlations were significant $(P<0.0001)$ between AUDPC-DI and MDIs of 9 and 14 DAI (the 2014 to 2015 experiment) or 15 DAI (the 2016 to 2017 experiment) of samplings for the 2 years' data (Fig. 3), and the correlation coefficients were $0.8192,0.8968,0.8978$, and 0.9444 , respectively (Fig. 3). The correlation between AUDPC-DI and MDI of 1 DAI sampling was not significant at $\alpha=0.05$, whereas that between AUDPC-DI and MDI of 5 DAI sampling was significant at $\alpha=0.05$; however, the correlation coefficient was relatively low with data of the 2014 to 2015 experiment (data not shown).

\section{Discussion}

Based on the 2-year experiments, this study showed that the correlations between AUDPC-DI and MDIs of 9 or 14 to 15 DAI of samplings during the latent period were all significant. We also found that the resistance levels of cultivars (lines) evaluated based on AUDPC-DI data were highly consistent with those based on MDI data. Most of the assays relying on visual assessment of the symptoms, lesion measurement, or ITs are time consuming and labor intensive, especially for larger numbers of tested materials. Comparatively, the MDI data, which could be obtained in a short amount of time and efficiently, can be used to estimate the possible resistance level to represent its dynamic expression in the fields. The molecular approach can likely replace the filed approaches for evaluation of varietal resistance to wheat stripe rust. We found that about 10 days after infection was the best time for leaf sampling to obtain MDI data. This study provided a new and efficient method for evaluation of varietal resistance to wheat stripe rust.

It was less accurate when using MDI data to evaluate resistance levels for moderately resistant and moderately susceptible cultivars (lines). This was perhaps because of the fact that most adultresistant cultivars (lines) showed moderate resistance or high resistance based on AUDPC-DI data, and part of their resistance could be induced by high temperature. Thus, $P$. striiformis f. sp. tritici may be developed in the plant tissues during the latent period when the temperature was relatively low, whereas the disease was suppressed later when the temperature was high in the fields, which was called high-temperature adult plant resistance (Chen 2013). Moreover, it may help to find more cultivars (lines) with horizontal resistance by comparing resistance of cultivars (lines) with the methods of AUDPC-DI and MDI.

The sampling times to test latent infections in this study were arranged four times after inoculation in 2015. The correlations between AUDPC-DI and MDIs of 9 and 14 DAI of samplings were both significant, whereas that of 1 DAI of sampling was not significant at $P=0.05$, and that of 5 DAI sampling was significant but with a low correlation coefficient. The development of $P$. striiformis $\mathrm{f}$. $\mathrm{sp}$. tritici inside wheat leaves during the latent period may be faster during 5 to 9 days after infection than in the earlier stage. Thus, increasing the number of samples in the later latent period could help us obtain a more accurate evaluation of host resistance.

The developments of $P$. striiformis $\mathrm{f}$. sp. tritici in different cultivars during the latent period were monitored by qPCR assay. The trend of MDIs was exponentially increased in susceptible cultivars (lines) during the sampling period, whereas the flexible trend was shown in resistant cultivars (lines). Furthermore, in these resistant cultivars (lines), the disease development curves of $P$. striiformis $\mathrm{f}$. sp. tritici could be helpful to distinguish different resistance types of cultivars (lines), such as those resistant to initial infection or those resistant to pathogen development inside leaf tissues. More accurate experiments to determine such resistance mechanisms during the latent period should be designed and tested in a future study.

Our results showed that most wheat cultivars (lines) used in the study exhibited all-stage resistance. Some wheat cultivars may still be resistant to $P$. striiformis f. sp. tritici races of CYR32 and CYR33 (popular in the past 15 years). Xue (2015) and Zeng (2011) both studied the resistance of wheat cultivars (lines) to $P$. striiformis $\mathrm{f}$. sp. tritici in the different wheat-producing areas, and the results showed that most cultivars (lines) planted in Longnan and Sichuan areas were resistant to $P$. striiformis f. sp. tritici. The study of Zeng (2011) showed that $47.8 \%$ of 69 cultivars (lines) showed allstage resistance, agreeing with the results of our study.

This research classified 100 cultivars (lines) into four groups based on resistance levels using cluster analysis with data of AUDPC-DI The results of classification were in accordance with ITs in general. However, for a few cultivars (lines), the results using AUDPC-DI data showed high resistance, where the results using ITs showed susceptibility, such as Chuannong 19 and Mianmai 168. The development of disease is a progress of interaction between host and pathogen. Also, wheat stripe rust is a polycyclic disease, and $P$. striiformis f. sp. tritici could infect wheat many times during the growing season. IT is a qualitative sign and relatively stable in the environment, whereas area under the disease progress curve (AUDPC) is a quantitative sign that could represent disease development in the whole epidemic period (Zeng et al. 1981a). The combination of methods using IT and AUDPC could obtain overall resistance evaluation of the tested cultivars.

qPCR was used to efficiently identify the host resistance during the interactions between host and pathogen. However, previous research paid attention to seeding stage and evaluated few varieties (Jackson et al. 2006; Yang et al. 2008). In comparison, this study focused on the feasibility and high efficiency of the methods of qPCR to identify the resistance of large numbers of samples combined with field experiments. Moreover, the method may be applied in evaluation of resistance in other pathosystems.

\section{Literature Cited}

Brouwer, M., Lievens, B., Van Hemelrijck, W., Van den Ackerveken, G., Cammue, B. P. A., and Thomma, B. P. H. J. 2003. Quantification of disease progression of several microbial pathogens on Arabidopsis thaliana using real-time fluorescence PCR. FEMS Microbiol. Lett. 228: $241-248$

Chen, X. 2005. Epidemiology and control of stripe rust [Puccinia striiformis $f . s p$. tritici] on wheat. Can. J. Plant Pathol. 27:314-337.

Chen, X. 2013. Review article: High-temperature adult-plant resistance, key for sustainable control of stripe rust. Am. J. Plant Sci. 4:608-627.

Cullen, D. W., Lees, A. K., Toth, I. K., and Duncan, J. M. 2001. Conventional PCR and real-time quantitative PCR detection of Helminthosporium solani in soil and on potato tubers. Eur. J. Plant Pathol. 107:387-398. 
Enjalbert, J., Duan, X., Giraud, T., Vautrin, D., de Vallavieille-Pope, C., and Solignac, M. 2002. Isolation of twelve microsattelite loci, using an enrichment protocol, in the phytopathogenic fungus Puccinia striiformis $\mathrm{f}$. sp. tritici. Mol. Ecol. Notes 2:563-565.

Filion, M., St-Arnaud, M., and Jabaji-Hare, S. H. 2003. Direct quantification of fungal DNA from soil substrate using real-time PCR. J. Microbiol. Methods 53:67-76.

Gachon, C., and Saindrenan, P. 2004. Real-time PCR monitoring of fungal development in Arabidopsis thaliana infected by Alternaria brassicicola and Botrytis cinerea. Plant Physiol. Biochem. 42:367-371.

Huang, Q., Li, X., Chen, W., Xiang, Z., Zhong, S., Chang, Z., Zhang, M., Zhang, H., Tan, F., and Ren, Z. 2014. Genetic mapping of a putative Thinopyrum intermedium-derived stripe rust resistance gene on wheat chromosome $1 \mathrm{~B}$. Theor. Appl. Genet. 127:843-853.

Jackson, E. J., Avant, J. B., Overturf, K. E., and Bonman, J. M. 2006. A quantitative assay of Puccinia coronata f. sp. avenae DNA in Avena sativa. Plant Dis. 90:629-636.

Jones, J. D. G., and Dangl, J. L. 2006. The plant immune system. Nature 444: 323-329.

Liu, B., Liu, T., Zhang, Z., Jia, Q., Wang, B., Gao, L., Peng, Y., Jin, S., and Chen, W. 2017. Discovery and pathogenicity of CYR34, a new race of Puccinia striiformis f. sp. tritici in China. Acta Phytopathologica Sin. 47:681-687.

Liu, T., Peng, Y., Chen, W., and Zhang, Z. 2010. First detection of virulence in Puccinia striiformis f. sp. tritici in China to resistance genes Yr24 (=Yr26) present in wheat cultivar chuanmai 42. Plant Dis. 94:1163.

Luo, Y., Gao, W., Doster, M., and Michailides, T. J. 2009. Quantification of conidial density of Aspergillus flavus and A. parasiticus in soil from almond orchards using real-time PCR. J. Appl. Microbiol. 106:1649-1660.

Luo, Y., Ma, Z., Reyes, H. C., Morgan, D. P., and Michailides, T. J. 2007. Quantification of airborne spores of Monilinia fructicola in stone fruit orchards of California using real-time PCR. Eur. J. Plant Pathol. 118:145-154.

McNeal, F. H., Konzak, C. F., Smith, E. P., Tate, W. S., and Russell, T. S. 1971. A uniform system for recording and processing cereal research data. USDA ARS. 42:34-121

McNeil, M., Roberts, A. M. I., Cockerell, V., and Mulholland, V. 2004. Real-time PCR assay for quantification of Tilletia caries contamination of UK wheat seed. Plant Pathol. 53:741-750.

Mercado-Blanco, J., Collado-Romero, M., Parrilla-Araujo, S., Rodríguez-Jurado, D., and Jiménez-Díaz, R. M. 2003. Quantitative monitoring of colonization of olive genotypes by Verticillium dahliae pathotypes with real-time polymerase chain reaction. Physiol. Mol. Plant Pathol. 63:91-105.

Pan, J., Luo, Y., Huang, C., Sun, Z., Zhao, L., Yan, J., and Ma, Z. 2010. Quantification of latent infections of wheat stripe rust by using real-time PCR. Acta Phytopathologica Sin. 40:504-510.

Pan, Y., Gu, Y., Luo, Y., and Ma, Z. 2016. Establishment and application of duplex real-time PCR quantitative determination method on latent infection of wheat stripe rust. Acta Phytopathologica Sin. 46:485-491.

Qi, M., and Yang, Y. 2002. Quantification of Magnaporthe grisea during infection of rice plants using real-time polymerase chain reaction and northern blot/ phosphoimaging analyses. Phytopathology 92:870-876.

Sandberg, M., Lundberg, L., Ferm, M., and Yman, I. M. 2003. Real-time PCR for the detection and discrimination of cereal contamination in gluten free foods. Eur. Food Res. Technol. 217:344-349.

Schweigkofler, W., O'Donnell, K., and Garbelotto, M. 2004. Detection and quantification of airborne conidia of Fusarium circinatum, the causal agent of pine pitch canker, from two California sites by using a real-time PCR approach combined with a simple spore trapping method. Appl. Environ. Microbiol. 70:3512-3520.

Shang, H., Ren, W., and Wang, S. 1990. The discussion of a grading standard of wheat stripe rust severity. Plant Prot. 16:31.

Stubbs, R. W. 1985. Stripe rust. Pages 61-101 in: The Cereal Rusts II. A. P. Roelfs and W. R. Bushnell, eds. Academic Press, Orlando, FL.

Stubbs, R. W. 1988. Pathogenicity analysis of yellow (stripe) rust of wheat and its significance in a global context. Pages 23-38 in: Breeding Strategies for Resistance to the Rusts of Wheat. N. W. Simmonds and A. Rajaram, eds. CIMMYT, Texcoco, Mexico.

Vandemark, G. J., and Barker, B. M. 2003. Quantifying Phytophthora medicaginis in susceptible and resistant alfalfa with a real-time fluorescent PCR assay. J. Phytopathol. 151:577-583.

Waalwijk, C., van der Heide, R., de Vries, I., van der Lee, T., Schoen, C., Corainville, G. C., Häuser-Hahn, I., Kastelein, P., Köhl, J., Lonnet, P., Demarquet, T., and Kema, G. H. J. 2004. Quantitative detection of Fusarium species in wheat using TaqMan. Eur. J. Plant Pathol. 110:481-494.

Wan, A., Chen, X., and He, Z. 2007. Wheat stripe rust in China. Aust. J. Agric. Res. 58:605-619.

Wan, A., Zhao, Z., Chen, X., He, Z., Jin, S., Jia, Q., Yao, G., Yang, J., Wang, B., Li, G., Bi, Y., and Yuan, Z. 2004. Wheat stripe rust epidemic and virulence of Puccinia striiformis f. sp. tritici in China in 2002. Plant Dis. 88:896-904

Wellings, C. R. 2007. Puccinia striiformis in Australia: A review of the incursion, evolution, and adaptation of stripe rust in the period 1970-2006. Aust. J. Agric Res. 58:567-575.

Wilhelm, J., and Pingoud, A. 2003. Real-time polymerase chain reaction. ChemBioChem 4:1120-1128.

Xiao, Y., Zeng, S., Zhang, W., and Wang, P. 1983. SIMYR-A simple simulation model of wheat stripe rust epidemiology. Acta Phytopathologica Sin. 13:1-13.

Xue, R., Zhu, Z., Huang, Y., Wang, X., Wang, L., and Wang, S. 2012. Quantification of Fusarium oxysporum f. sp. phaseoli detected by real-time quantitative PCR in different common beans cultivars. Acta Phytopathologica Sin. 38:791-799.

Xue, W. 2015. Establishment and application of evaluation system for the resistance of wheat to stripe rust. Dissertation. Northwest A\&F University, Yangling, China.

Yan, J., Luo, Y., Chen, T., Huang, C., and Ma, Z. 2012. Field distribution of wheat stripe rust latent infection using real-time PCR. Plant Dis. 96:544-551.

Yang, J., Fang, Z., Zhang, M., Xu, F., Wang, W., Xie, L., Lin, Q., Wu, Z., and Xie, L. 2008. Detection on different resistance of two rice varieties against rice stripe virus in real-time PCR. J. Sourth China Agric. Univ. 29:25-28.

Zadoks, J. C., Chang, T. T., and Konzak, C. F. 1974. A decimal code for the growth stages of cereals. Weed Res. 14:415-421.

Zeng, Q. 2011. Analysis of resistance genes to stripe rust in current winter wheat cultivars in China. Dissertation. Northwest A\&F University, Yangling, China.

Zeng, S., and Luo, Y. 2006. Long-distance spread and interregional epidemics of wheat stripe rust in China. Plant Dis. 90:980-988.

Zeng, S., Wang, P., and Zhang, W. 1981a. Relative resistance index-one of improvement of the evaluation of resistance to Pst of wheat. Acta Phytopathologica Sin. 11:7-12.

Zeng, S., Zhang, W., and Xiao, Y. 1981b. A preliminary study on wheat stripe rust computer simulation. J. Beijing Agric. Univ. 7:1-12.

Zeng, X., Luo, Y., Zheng, Y., Duan, X., and Zhou, Y. 2010. Detection of latent infection of wheat leaves caused by Blumeria graminis f. sp. tritici using nested PCR. J. Phytopathol. 158:227-235. 